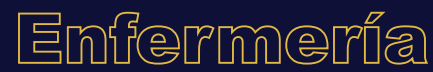

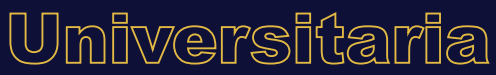

\section{Relación entre automanejo y síntomas depresivos en personas con diabetes e hipertensión}

\author{
Relationship between self-management and \\ depressive symptoms in persons with diabetes \\ and hypertension
}

\section{Relação entre automanejo e sintomas depressivos em pessoas com diabetes e hipertensão}

\section{M.S. De León-Ramírez ${ }^{a *}$, M.I. Peñarrieta-de Córdova ${ }^{b_{2}}$,} R. León-Hernández ${ }^{\mathrm{c}}$, T. Gutiérrez-Gómez ${ }^{\mathrm{d} 2}$, M.S. Piñones-Martínez ${ }^{\mathrm{e} 2}$, L.M. Ouintero-Valle ${ }^{\mathrm{f}}$

ORCID:

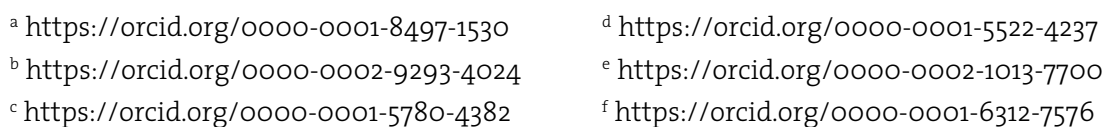

${ }^{1}$ Universidad Autónoma del Estado de Hidalgo, Escuela Superior de Huejutla, Huejutla, Hidalgo, México ${ }^{2}$ Universidad Autónoma de Tamaulipas, Facultad de Enfermería, Tampico, Tamaulipas, México 3Universidad Autónoma Tamaulipas, Facultad de Enfermería, Catedrático CONACYT, Tampico, Tamaulipas, México

Recibido: 8 junio 2019 / Aceptado: 25 noviembre 2019

\section{RESUMEN}

Introducción: Las personas con diabetes e hipertensión experimentan con mayor frecuencia sintomatología depresiva, lo cual contribuye a un inadecuado automanejo de la enfermedad, que implica tareas como: la toma de la medicación, asistir a las consultas médicas, el conocimiento de signos y sintomas, además de la toma de decisiones.

Objetivo: Por lo anterior, el objetivo de este trabajo es identificar la relación entre automanejo en general y sus dimensiones con síntomas depresivos en personas con diabetes e hipertensión.

Método: Estudio transversal-correlacional con $\mathrm{n}=205$ pacientes con diagnóstico de diabetes (100) e hipertensión (105). Muestreo no probabilístico por conveniencia. Se utilizó la estadística descriptiva y r de Pearson. Se aplicaron los instrumentos Partners in Health (PIH) y The Patient Health Questionnaire (PHO-8).

*Autor de correspondencia. Correo electrónico: deleon281091@gmail.com https://doi.org/10.22201/eneo.23958421e.2020.1.681

1665-7063/@ 2020 Universidad Nacional Autónoma de México, Escuela Nacional de Enfermería y Obstetricia. Este es un artículo Open Access bajo la licencia CC BY-NC-ND (http://creativecommons.org/licenses/by-nc-nd/4.o/). 
Resultados: Se encontró correlación estadísticamente significativa entre la sintomatología depresiva y el automanejo ( $\mathrm{r}=-0.308 \rho<0.001)$.

Discusión y Conclusiones: Se concluye que la sintomatología depresiva se relaciona con el automanejo de la enfermedad de la siguiente manera: a mayor automanejo menor sintomatología de depresión, o a mayor sintomatología depresiva menor automanejo; dicha relación confirma que ambas variables se afectan mutuamente y/o mantienen una relación estrecha. Palabras clave: Autocuidado; enfermedades crónicas; sintomatología depresiva; diabetes mellitus; hipertensión; México.

\section{ABSTRACT}

Introduction: Persons with diabetes and hypertension frequently suffer from depression as well, a situation which contributes to an inadequate management of the condition in terms of medication, medical consultations, signs, and decision making.

Objective: To identify the relationship between general self-management and depression symptoms in persons with diabetes and hypertension.

Method: This is a transversal and correlational study with a sample of 205 patients, 100 with a main diagnosis of diabetes, and 105 with a main diagnosis of hypertension. The sampling process was non-probabilistic and by convenience. Descriptive statistics, including Pearson's $r$ were calculated. The Partners in Health (PIH) and Patient Health Questionnaire (PHO-8) instruments were administered.

Results: A statistically significant correlation between depression symptoms and diabetes and hypertension self-management was found $(\mathrm{r}=-0.308 \rho<0.001)$.

Discussion and conclusions: Depression symptoms were related to how diabetes and hypertension are self-managed in a way that, the more self-management, the less depression symptoms, or the more depression symptoms, the less self-management.

Keywords: Self-care; chronic diseases; depressive symptomatology; diabetes mellitus; hypertension; Mexico.

\section{RESUMO}

Introdução: As pessoas com diabetes e hipertensão experimentam com maior frequência a sintomatologia depressiva, o qual contribui a um inadequado automanejo da doença, que envolve tarefas como: a toma da medicação, assistir às consultas médicas, o conhecimento de signos e sintomas, além da toma de decisões.

Objetivo: Pelo anterior, o objetivo deste trabalho é identificar a relação entre automanejo em geral e suas dimensões com sintomas depressivos em pessoas com diabetes e hipertensão. Método: Estudo transversal-correlacional com $\mathrm{n}=205$ pacientes com diagnóstico de diabetes (100) e hipertensão (105). Amostragem não probabilística por conveniência. Utilizou-se a estatística descritiva er de Pearson. Aplicaram-se os instrumentos Partners in Health (PIH) e The Patient Health Questionnaire (PHQ-8).

Resultados: Encontrou-se correlação estatisticamente significativa entre a sintomatologia depressiva e o automanejo ( $\mathrm{r}=-0.308 \rho<0.001)$.

Discussão e Conclusões: Conclui-se que a sintomatologia depressiva relaciona-se com o automanejo da doença da seguinte maneira: a maior automanejo, menor sintomatologia de depressão, ou a maior sintomatologia depressiva, menor automanejo; esta relação confirma que ambas variáveis afetam-se mutuamente e/ou mantem uma relação estreita.

Palavras chave: Autocuidado; doenças crônicas; sintomatologia depressiva; diabetes mellitus; hipertensão; México. 


\section{INTRODUCCIÓN}

Las enfermedades crónicas no transmisibles, como la diabetes y la hipertensión, representan uno de los mayores retos para los sistemas de salud. Se calcula que alrededor de 285 millones de personas en el mundo padecen alguna de estas enfermedades y esta cifra aumentará a 439 millones para 20301. En 2000, en México la diabetes fue la primera causa de muerte en mujeres y la segunda en hombres²; su prevalencia incrementó de $9.2 \mathrm{al}$ 9.4\% del $2012 \mathrm{al} \mathrm{20163}$. Por otra parte, la prevalencia nacional de hipertensión reportada en 2016 fue de $25.5 \%$, y cabe destacar que el 40\% no tenía conocimiento que vivía con esta enfermedad3. En términos generales, más del 50\% de la población mexicana sufre por lo menos una enfermedad crónica y más de la mitad lo desconoce $4^{4-6}$.

La situación anterior es preocupante si se considera que las enfermedades crónicas afectan el bienestar y calidad de vida de quienes las padecen, limitan a las personas en su cotidianidad y sus prácticas culturales ${ }^{7}$ además, se sabe que quienes las padecen experimentan estados emocionales negativos como la sintomatología depresiva, frustración e incertidumbre, lo cual se relaciona significativamente con un peor estado de salud 8 .

En estudios con personas que padecen diabetes se han reportado porcentajes de sintomatología depresiva que van desde $6 \%$ a 50\%.9-16; se asume así que este grupo de personas tiene mayor probabilidad de presentar sintomatología depresiva ${ }^{10,11}$. En términos generales, existe evidencia suficiente de la relación entre sintomatología depresiva y algún indicador de diabetes (HbA1c y glucemia en ayunas) $)^{10-13,17-19}$.

Con respecto a la hipertensión, es sabido que las personas con esta enfermedad experimentan emociones fuertes que aumentan el riesgo de padecer sintomatología depresiva ${ }^{20}$. También, se ha documentado que la sintomatología depresiva se asocia a una inadecuada adherencia al tratamiento cardiovascular y mal pronóstico de la enfermedad ${ }^{21}$. Los porcentajes de sintomatología depresiva en estudios con este grupo de personas oscilan entre 4 y $58 \%{ }^{20-25}$.

Se ha demostrado que las personas que desconocen cursar un padecimiento crónico tienen menor riesgo a desarrollar síntomas depresivos que quienes lo conocen, o no han sido previamente diagnosticadas ${ }^{22}$. De igual forma, diversos estudios documentan la relación entre sintomatología depresiva y presión arterial alta20,23,24,26.

No cabe duda de que tanto la diabetes como la hipertensión representan un grave problema de salud para quienes las padecen; sin embargo, se sabe que sus complicaciones y la sintomatología depresiva que las acompaña se pueden prevenir con un manejo adecuado. Por ello, la Organización Panamericana de Salud (OPS) promueve el apoyo al automanejo como un elemento clave del modelo de cuidados crónicos ${ }^{27}$. El automanejo es conceptualizado como la habilidad y capacidad del paciente para comprender la naturaleza de su condición, gestionar y organizar acceso a elementos de su cuidado, reconocer las señales de alarma para evitar complicaciones innecesarias y la manera de mantener o recuperar la estabilidad emocional y psicológica ${ }^{28}$.

El apoyo al automanejo prueba ser eficaz por integrar la atención y el tratamiento de las enfermedades crónicas y trastornos mentales en servicios de primer nivel. ${ }^{27}$ Además, está demostrado el beneficio del automanejo en personas con enfermedades crónicas que cursan sintomatología depresiva, ya que han utilizado una amplia gama de estrategias de automanejo para su recuperación²7,29,30.

En confirmación de lo anterior, diversos estudios han documentado el efecto de los programas de automanejo sobre sintomatología depresiva en grupos con diferentes padecimientos. Un estudio realizado en personas con artritis reumatoide encontró una reducción de la sintomatología depresiva después de una intervención de automanejo ${ }^{31}$. Un programa de este tipo aplicado a una muestra 
de personas con diabetes reportó mejoras significativas en sintomatología depresiva ${ }^{32}$; resultados similares también fueron reportados en un metaanálisis con personas con insuficiencia renal, se encontraron efectos positivos en sintomatología depresiva ${ }^{33}$. Del mismo modo, en otro estudio realizado con personas con enfermedades mentales se reportaron mejoras estadísticamente significativas en el indicador de depresión ${ }^{34}$. Así también, en un estudio en pacientes con cáncer de mama se detectó una reducción de la sintomatología depresiva ${ }^{35}$.

Aunado a lo anterior, un metaanálisis refiere que el automanejo mejora el estado emocional y psicológico de las personas que padecen una enfermedad crónica, ayuda a responder de manera positiva a las variaciones en el estado de salud, sobrellevar los sentimientos relacionados a la progresión de la enfermedad, la exacerbación de los síntomas y además favorece el establecimiento de metas del automanejo ${ }^{36}$.

Además de estos estudios que documentan los efectos favorables del automanejo, también se precisan los efectos de su ausencia; a partir de una metasíntesis con muestras de adultos mayores con enfermedad crónica, se identificó que la sintomatología depresiva es un factor que puede afectar el automanejo de la enfermedad ${ }^{37}$. De igual forma, se ha identificado correlación significativa entre depresión y automanejo en personas con diabetes ${ }^{38}$, así como una relación causal entre el estado de ánimo deprimido y deficiente automanejo de esta enfermedad ${ }^{39}$.

Entre los avances de investigación en países latinoamericanos, se reportó en un estudio en personas peruanas con enfermedades crónicas (diabetes, hipertensión y cáncer) un deficiente automanejo en todas sus dimensiones: conocimiento, adherencia y manejo de signos/síntomas; además, un $40 \%$ de la muestra presentó problemas asociados a la sintomatología depresiva: sentirse desanimado, poca energía, dificultad en concentrarse y desinterés en hacer cosas ${ }^{40}$. En México, se ha documentado la relación entre automanejo y funcionalidad familiar ${ }^{41}$, y la sintomatología depresiva como predictor del comportamiento de automanejo en personas con diabetes, hipertensión y obesidad ${ }^{42}$.

Si bien es cierto que se tiene un avance en el conocimiento del automanejo y su interacción con la sintomatología depresiva, la de México es insuficiente. En el presente estudio desde el ámbito de la enfermería, interesa, además de aportar mayor evidencia a dicha asociación y la relación entre las dimensiones del automanejo y la sintomatología depresiva, generar conocimiento a fin de fundamentar propuestas específicas que contribuyan a disminuir los efectos negativos de las enfermedades crónicas. Por lo expuesto, el objetivo de este trabajo es identificar la relación entre automanejo en general, y sus dimensiones, con la sintomatología depresiva en personas con diabetes e hipertensión.

\section{MÉTODOS}

El presente estudio tuvo un diseño transversal / correlacional, en el cual se consideraron las variables en estudio: automanejo y sintomatología depresiva.

Se realizó un muestreo por conveniencia, la muestra quedó constituida por 205 participantes, 100 con diagnóstico de diabetes y 105 con diagnóstico de hipertensión, pertenecientes a 7 centros de salud de la Jurisdicción Sanitaria No.2 Tampico, de la Secretaría de Salud del estado de Tamaulipas, México. Se seleccionaron personas mayores de 18 años, con un diagnóstico previo superior a 3 meses y que firmaran el consentimiento para participar en el estudio. Se excluyeron del análisis a mujeres embarazadas o personas con un padecimiento médico o mental que interfiriera con los fines de esta investigación. 
Para describir los datos sociodemográficos de los participantes, se utilizó una cédula de identificación que incluyó los datos de edad, sexo, estado civil y años de estudio.

Para medir la variable automanejo se utilizó el instrumento Partners In Health (PIH), validado en México; consta de 12 reactivos con formato de respuesta visual-numérico que va de o a 8. El rango de puntajes se homogeniza a un puntaje entre o y 100 con una regla de tres, una puntuación alta indica un mejor automanejo. La validez del constructo se obtuvo mediante un análisis factorial que arrojó 3 dimensiones: conocimiento (reactivos 1 y 2), adherencia (reactivos 3, 4, 5, 6, 7 y 8) y manejo de signos y síntomas (reactivos 9,10,11 y 12). También, se han conseguido puntos de corte para obtener niveles de automanejo. Su confiabilidad se calculó mediante alfa de Cronbach=0.88 43 .

Los sintomas depresivos se evaluaron mediante el instrumento The Patient Health Questionnaire (PHQ-8). El instrumento fue desarrollado por Kronke et al.; comprende 8 de los 9 criterios del Manual Diagnóstico y Estadístico de los Trastornos Mentales, cuarta edición ${ }^{44}$. Indica los síntomas experimentados durante 2 semanas antes de la entrevista 45 .

El instrumento está formado por 8 reactivos, con formato de respuesta tipo Likert; cada reactivo tiene puntuaciones de o (ningún día) a 3 (casi todos los días). El rango de puntajes se obtiene mediante la suma de cada reactivo que va de un rango de o a 24 puntos. Las puntuaciones se categorizan del siguiente modo: sin síntomas depresivos (o-4 puntos), síntomas leves (5-9 puntos), síntomas moderados (10-14), sintomas moderadamente severos (15-19 puntos) y síntomas graves (20-24 puntos). La confiabilidad en México se obtuvo con alpha de Cronbach =79 y el coeficiente de correlación interclase (test-retest) $=0.85^{43}$.

Para el procedimiento de recolección de datos se formaron equipos de trabajo constituidos por docentes y estudiantes del último año de la Licenciatura de la Facultad de Enfermería Tampico de la Universidad Autónoma de Tamaulipas; previamente todo el equipo recibió una capacitación para aplicar los instrumentos, el reclutamiento de participantes y levantamiento de datos que se realizó en los centros de salud. El estudio se apegó a la Ley General de Salud en Materia de Investigación para la Salud; se sometió al dictamen de las comisiones de Investigación y Ética de la Facultad de Enfermería Tampico de la Universidad Autónoma de Tamaulipas y además se obtuvo la autorización de la Secretaría de Salud del Estado de Tamaulipas.

Con relación a las técnicas de procesamiento y análisis de datos, se utilizó el Paquete Estadístico para la Ciencias Sociales (SPSS por sus siglas en inglés) versión 23 para Windows. Se aplicó estadística descriptiva con análisis de frecuencias y porcentajes; además, se utilizó el coeficiente de correlación $r$ de Pearson ya que las variables sintomatología depresiva y automanejo con sus 3 dimensiones presentaron parámetros de normalidad mediante la prueba Kolmogorov Smirnov ( $p>$.05).

\section{RESULTADOS}

En la Tabla 1 se puede observar que la media de edad de la muestra fue de 58 años; con predominio de mujeres (75\%). La media de años de estudios fue de 7 y más de la mitad reportaron ser casados (67\%).

Se aplicó una prueba de comparación (t de Student para muestras independientes) para explorar diferencias en sintomatología depresiva y automanejo, entre los subgrupos de participantes con diabetes e hipertensión. Los resultados no mostraron diferencias significativas en ninguno de los indicadores antes mencionados, por tanto, los resultados posteriores se presentan sin hacer distinción entre los subgrupos.

Con relación a la presencia de sintomatología depresiva destaca que más de la mitad de la muestra total (61\%) puntuó en la categoría sin síntomas. Sin embargo, un 17.5\% presenta síntomas moderados a severos (Tabla 2). 
Tabla 1. Aspectos demográficos de la población de estudio $(\mathbf{N}=\mathbf{2 0 5})$

\begin{tabular}{|c|c|c|c|}
\hline \multirow{2}{*}{ Aspectos demográficos } & $F$ & \multicolumn{2}{|c|}{$\%$} \\
\hline & $(n=205)$ & \multicolumn{2}{|c|}{$(100)$} \\
\hline Edad en años & $\dot{x}=58.6( \pm 10.7)$ & Min:27 & Max: 82 \\
\hline Educación (años de estudio) & $\dot{x}=7( \pm 3.6)$ & Min: o & Max: 18 \\
\hline \multicolumn{4}{|l|}{ Género } \\
\hline Mujer & 153 & \multicolumn{2}{|c|}{75} \\
\hline Hombre & 52 & \multicolumn{2}{|c|}{25} \\
\hline \multicolumn{4}{|l|}{ Estado Civil } \\
\hline Casado & 138 & \multicolumn{2}{|c|}{67} \\
\hline Viudo & 18 & \multicolumn{2}{|c|}{9} \\
\hline Divorciado & 8 & \multicolumn{2}{|c|}{4} \\
\hline Soltero & 41 & \multicolumn{2}{|c|}{20} \\
\hline
\end{tabular}

Tabla 2. Sintomatología depresiva en la población de estudio

\begin{tabular}{lcc}
\hline Trastorno depresivo & F & \% \\
\hline Sin síntomas & 125 & 61.0 \\
\hline Síntomas leves & 45 & 22.0 \\
\hline Síntomas moderados & 18 & 8.8 \\
\hline Síntomas moderadamente severos & 14 & 6.8 \\
\hline Síntomas Severos & 3 & 1.5 \\
\hline Total & 205 & 100 \\
\hline
\end{tabular}

El índice general del comportamiento de automanejo presentó una media de 79, en la que 100 indica un adecuado automanejo de la enfermedad crónica. En relación con las dimensiones (conocimiento de la enfermedad, manejo de signos y síntomas y adherencia), la que presentó menor puntaje fue la de conocimiento con una media de 69. (Tabla 3).

Tabla 3. Automanejo de la enfermedad crónica ( $\mathrm{N}=\mathbf{2 0 5})$

\begin{tabular}{lcccc}
\hline Automanejo & Mínimo & Máximo & Media & Desv. Tip \\
\hline Índice General & 0 & 100 & 79.43 & 17.02 \\
\hline Conocimiento & $\mathrm{O}$ & 100 & 69.20 & 23.90 \\
\hline Manejo de signos y síntomas & $\mathrm{O}$ & 100 & 81.50 & 17.09 \\
\hline Adherencia & $\mathrm{O}$ & 100 & 83.00 & 16.39 \\
\hline
\end{tabular}

Para alcanzar el objetivo de investigación se aplicó un coeficiente de correlación r de Pearson. Los datos muestran una correlación significativa negativa entre la sintomatología depresiva (puntaje total) y el índice general de automanejo.

Igualmente se encontraron correlaciones significativas negativas en las tres dimensiones del automanejo: 1) conocimiento, 2) manejo de signos y síntomas y 3) adherencia, todas con sintomatología depresiva. La dirección de las correlaciones se puede interpretar como: a mayor sintomatología depresiva menor automanejo o a la inversa (tabla 4). 
Tabla 4. Correlación entre sintomatologia depresiva e indicadores de Automanejo

\begin{tabular}{lccccc}
\hline & & $\begin{array}{c}\text { Índice general } \\
\text { de automanejo }\end{array}$ & Conocimiento & $\begin{array}{c}\text { Manejo Signos y } \\
\text { sintomas }\end{array}$ & Adherencia \\
\hline $\begin{array}{l}\text { Sintomatología } \\
\text { depresiva }\end{array}$ & r de Pearson & $-.308^{* *}$ & $-.195^{* *}$ & $-.444^{* *}$ & $-.161^{*}$ \\
\hline & Sig. bilateral & .000 & .005 & .000 & .021 \\
\hline
\end{tabular}

**. Correlación es significativa al nivel 0.01 (bilateral)

*. Correlación es significativa al nivel 0.05 (bilateral

\section{DISCUSIÓN}

Es importante mencionar que en el presente estudio no se detectaron diferencias en automanejo y síntomas de depresión al comparar el subgrupo de personas con diabetes y el de personas con hipertensión; por lo anterior, los análisis se realizaron con la muestra en su totalidad.

El índice general del automanejo se encuentra en una media de 79, lo que indica que el automanejo en la muestra no es el óptimo; esto concuerda con estudios realizados en México en los que se han reportado índice general entre 74 y 81 en muestras de personas con diabetes, hipertensión y cáncer. ${ }^{41,42}$ Dichos resultados difieren a los encontrados en Perú, con una muestra similar de personas con las mismas enfermedades, en las que se detectó un índice general de automanejo más deficiente $(69)^{40}$.

En cuanto a las dimensiones del instrumento, manejo de signos y sintomas tuvo una puntuación de 81.5; de igual forma, un estudio mexicano presenta puntajes similares en esta dimensión (81) ${ }^{41}$, y a diferencia del patrón de automanejo en el estudio realizado en Perú, el puntaje en esta dimensión es menor $(70)^{40}$.

En la dimensión de adherencia se presentó la mejor puntuación media de 83. Al igual que los resultados anteriormente descritos, se encuentra similitud en los puntajes de México $(83)^{41}$ y un promedio menor en el estudio peruano (70 $4^{40}$. Las diferencias en el automanejo en los estudios en muestras mexicanas y peruanas pudieran atribuirse a las características socioculturales de estos diferentes países, o bien, a que en el estudio peruano se incluyeron personas con cáncer, que pudieron marcar la diferencia en el automanejo de la enfermedad.

Con relación a la presencia del trastorno depresivo, más de la tercera parte de la muestra presenta dichos síntomas (39\%): este resultado concuerda con la literatura internacional en la que se ha reportado presencia de síntomas depresivos entre el 4 y 58\% en personas con enfermedades crónicas como diabetes ${ }^{9-16}$ e hipertensión ${ }^{20,21-25}$. Cabe destacar que los síntomas severos de depresión fueron reportados únicamente por el 1.5\% de la muestra de este estudio, dato que concuerda con trabajos anteriores que reportan sintomatología severa en porcentajes mínimos (4 a 4.3\% ${ }^{20,25}$. Sin embargo, es importante recordar que el cursar enfermedades como diabetes e hipertensión, incrementa el riesgo de desarrollar sintomatología depresiva ${ }^{10,11,20,}$ que se asocia con un mal pronóstico de la enfermedad ${ }^{21}$.

El resultado de mayor relevancia fue la correlación entre el trastorno depresivo y el índice general de automanejo, negativa y estadísticamente significativa; esto es: a mayor sintomatología depresiva menor automanejo o viceversa. Estos resultados concuerdan con un estudio realizado con una muestra de personas con diabetes en el que se reportaron correlaciones entre sintomatología de depresión y diferentes indicadores de automanejo ${ }^{39}$, o bien, se ha identificado la sintomatología depresiva como predictor de automanejo ${ }^{42}$.

Si bien se tiene evidencia suficiente de la relación entre sintomatología depresiva con diabetes ${ }^{10-13,17-19}$ e hipertensión, ${ }^{20,23,24,26}$ es insuficiente el avance en el conocimiento de las relaciones entre el 
automanejo de estas enfermedades y los sintomas de depresión; por tanto, el aporte de este estudio responde al objetivo y la relevancia de la presente investigación.

Finalmente, es importante destacar que se tiene amplio conocimiento sobre el efecto positivo que tienen los programas de automanejo sobre los indicadores de depresión en personas con artritis, diabetes, hipertensión, insuficiencia renal y enfermedades mentales. ${ }^{18-21}$ Sin embargo, en contexto mexicano no se detectó literatura suficiente asociada a lo anterior; por tanto, el avance teórico empírico que antecede a las intervenciones y permite entender la relación entre estas variables es de suma importancia, ya que representa un sustento que puede ser un aporte para mejorar los programas de automanejo y, como consecuencia lógica, la salud de las personas con enfermedades crónicas.

\section{CONCLUSIONES}

A partir de lo anterior, se concluye que no existe diferencia en el comportamiento de automanejo y sintomatología en las muestras de personas con hipertensión y diabetes de este estudio. Además, se concluye que la sintomatología depresiva se relaciona con el automanejo de la enfermedad de la siguiente manera: a mayor automanejo menor sintomatología de depresión, o a mayor sintomatología depresiva menor automanejo; dicha relación concuerda con estudios anteriores y confirma que ambas variables se afectan mutuamente y/o mantienen una relación estrecha.

\section{RESPONSABILIDADES ÉTICAS}

Protección de personas y animales. Los autores declaran que los procedimientos realizados se ajustaron a la Declaración de Helsinki y a la Ley General de Salud de México en materia de investigación. Confidencialidad. Los autores declaran que han obtenido el consentimiento informado de los sujetos referidos en el estudio; asimismo los datos confidenciales fueron protegidos conforme al reglamento de la ley general de Salud en materia de investigación para la salud.

Financiamiento. Este proyecto fue financiado por CONACYT: Red temática de Automanejo en crónicos \#270842.

Agradecimientos. Agradecemos el apoyo técnico y económico en la realización del presente estudio a la Universidad Autónoma de Tamaulipas, Facultad de Enfermería campus Tampico, asimismo a la jurisdicción sanitaria No. II de la Secretaria de Salud de Tamaulipas.

\section{REFERENCIAS}

1. Organización Mundial de la Salud. Informe sobre la situación Mundial de las enfermedades no transmisibles. Ginebra: OMS; 2014. https://bit.ly/38WjJIT

2. Aguilar-Salinas C, Hernández-Jiménez S, Hernández-Ávila M, Hernández Ávila JE. Acciones para enfrentar a la diabetes. Documento de Postura. México, D.F: Academia Nacional de Medicina; 2015.

3. Secretaria de Salud-Instituto Nacional De Salud Pública. Encuesta Nacional de Salud y Nutrición de Medio Camino 2016. México: Secretaria de Salud; 2016. https://bit.ly/2S5pT2w

4. Olaiz-Fernández G, Rivera J, Shamah-Levy T, Rojas R, Villalpando-Hernández S, Hernández-Ávila M, et al. (Ed). Encuesta Nacional de Salud y Nutrición 2006. Cuernavaca, México: Instituto Nacional de Salud Pública; 2006. https://bit.ly/2GP8dmk

5. Olaiz G, Rojas R, Barquera S, Shamah T, Aguilar C, Cravioto P, et al. (Ed). Encuesta Nacional de Salud 2000. Tomo 2. La salud de los adultos. Cuernavaca, Morelos, México: Instituto Nacional De Salud Pública-Secretaria de Salud; 2003. https://bit.ly/2SbcS7X 
6. Gutiérrez J, Rivera-Dommarco J, Shamah-Levy T, Villalpando-Hernández S, Franco A, et al. Encuesta Nacional de Salud y Nutrición 2012. Resultados Nacionales. Cuernavaca, México: Instituto Nacional de Salud Pública; 2012. https://bit.ly/2uWxwOKK

7. Montalvo-Prieto A, Cabrera-Nanclares B, Quiñones-Arrieta S. Enfermedad crónica y sufrimiento: Revisión de literatura. Aquichan. 2012; 12(2): 134-43. https://doi.org/10.5294/aqui.2012.12.2.4

8. Orozco GAM, Castiblanco OL. Factores psicosociales e intervención psicológica en enfermedades crónicas no Transmisibles. Rev Colomb Psicol. 2015; 24(1): 203-17.

https://dx.doi.org/10.15446/rcp.v24n1.42949

9. Wilhem K, Reddy J, Crawford J, Robins L, Campell L, Proudfoot J. The Importance of screening for mild depression in adults with diabetes. Transl Biomed. 2017; 8(1).

https://doi.org/10.2167/2172-0479.1000101

10. Bădescu SV, Tătaru C, Kobylinka L, Georgescu EL, Zahiun DM, Zăgrean AM, et al. The association between diabetes mellitus and depression. J Med Life. 2016; 9(2): 120-5. https://bit.ly/2ROfIQN

11. Siddiqui S. Depression in type 2 diabetes mellitus: A brief review. Diabetes Metab Syndr. 2014; 8(1): 62-5. https://doi.org/10.1016/j.dsx.2013.06.010

12. Domingo AK, Asmal L, Seedat S, Esterhuizen TM, Laurence C, Volmink J. Investigating the association between diabetes mellitus, depression and psychological distress in a cohort of South African teachers. S Afr Med J. 2015; 105(12): 1057-6o. https://doi.org/10.7196/SAMJ.2015.v105i12.9843

13. Zhang Y, Ting RZ, Yang W, Jia W, Li W, Ji L, et al. Depression in chinese patients with type 2 diabetes: associations with hyperglucemia, hypoglucemia, and poor treat adherence. J Diabetes. 2015; 7(6): 8oo-8. https://doi.org/1111/1753-0407.12238

14. Olvera R, Fisher-Hoch SP, Williamson DE, Vatcheva KP, McCormick JB. Depression in Mexican Americans with diagnosed and undiagnosed diabetes. Psychol Med. 2016; 46(3); 637-43. https://doi.org/10.1017/So033291715002160

15. Lara MM, Jacobs EA, Escamilla M, Mendenhall E. Depression among diabetic women in urban centers in México and the United States of America: a comparative study. Rev Panam Salud Publica. 2014; 36(4):225-31.

16. Schmitt A, Reimer A, Kulzer B, Haak T, Gahr A, Hermanns N. Negative association between depression and diabetes control only when accompanied by diabetes-specific distress. J Behav Med. 2014; 38(3): 556-64. https://doi.org/10.1007/s10865-014-9604-3

17. Fisher L, Gonzalez JS, Polonsky WH. The confusing tale of depression and distress in patients with diabetes: a call for greater clarity and precision. Diabet Med. 2014; 31(7): 764-72. https://doi.org/10.1111/dme.12428

18. Yu M, Zhang X, Lu F, Fang L. Depression and risk for diabetes: A meta-analysis. Can J Diabetes. 2015; 39(4): 266-72. https://doi.org/10.1016/j.jcjd.2014.11.006

19. Chireh B, Li M, D’Arcy C. Diabetes increases the risk of depression: A systematic review, metaanalysis and estimates of population attributable fractions based on prospective studies. Prev Med Rep. 2019; 14: 100822. https://doi.org/10.1016/j.pmedr.2019.100822

20. Kretchy IA, Owusu-Daaku FT, Danquah SA. Mental Health in hypertension: assessing symptoms of anxiety, depression and stress on anti-hypertensive medication adherence. Int J Ment Health Syst. 2014; 8(25). https://doi.org/10.1186/1752-4458-8-25

21. Cohen BE, Edmondson D, Kronish IM. State of the Art Review: Depression, Stress, Anxiety, and cardiovascular disease. Am J Hypertens. 2015; 28(11): 1295-302. https://doi.org/10.1093/ajh/hpvo47 
22. Rantanen AT, Korkeila JJA, Loyttyniemi E, Saxén U, Korhonen PE. Awareness of hypertension and depressive symptoms: a cross-sectional study in a primary care population. Scand J Prim Health Care. 2018; 36(3): 323-8. https://doi.org/10.1080/02813432.2018.1499588

23. Xue K, Chen S, Bogner HR, Tang W, Li L, Conwell Y. The prevalence of depressive symptoms among older patients with hypertension in rural China. Int J Geriatr Psychiatry. 2017; 32(12): 1411-7. https://doi.org/10.1002/gps.4628

24. Shoaib-Hamrah $M$, Hassan-Hamrah $M$, Ishii H, Suzuki S, Hussain-Hamrah M, Edris-Hamrah A, et al. Anxiety and depression among hypertensive outpatients in Afghanistan: A Cross-Sectional Study in Andkhoy City. Int J Hypertens. 2018; 8. https://doi.org/10.1155/2018/8560835

25. Alkhathami A, Alamin M, Alqahtani A, Alsaeed W, Alkhathami M, Al-Dhafeeri A. Depression and anxiety among hypertensive and diabetic primary health care patients. Could patients' perception of their diseases control be used as a screening tool? Saudi Med J. 2017; 38(6): 621-8. https://doi.org/10.15537/smj.2017.6.17941

26. Liu MY, Li N, Li W, Khan H. Association between psychosocial stress and hypertension: a systematic. Review and meta-analysis. Neurol Res. 2017; 39(6): 573-80. https://doi.org/10.1080/01616412.2017

27. Organización Panamericana de la Salud. Cuidados Innovadores para las condiciones crónicas: Organización y prestación de atención de alta calidad a las enfermedades crónicas no transmisibles en las Américas. Washington, D.C: OPS; 2013.

28. Lawn S, Battersby M, Harvey P, Ackland A. A behavioural therapy approach to self-management: The Flinders Program. Diabetes Voice. 2009; 54: 30-2. https://bit.ly/2uXenhC

29. Loring K, Ritter P, Jacquez A. Outcomes of border health Spanish/English chronic disease selfmanagement programs. Diabetes educ. 2005; 31(3):404-9. http://dx.doi.org/10.1177/0145721705276574

30. Van Grieken R, Van Tricht M, Koeter M, Van den Brink W, Schene AH. The use and helpfulness of self-management strategies for depression: The experiences of patients. Plos One. 2018; 13(12):1-12. https://dx.doi.org/10.1371/journal.pone.0209109

31. Loring K, Mazonson P, Holman H. Evidence suggesting that health education for self-management in patients with chronic arthritis has sustained health benefits while reducing health care costs. Arthritis Rheum. 1993; 36(4): 439-46. https://dx.doi.org/10.1002/art.1780360403

32. Loring K, Ritter P, Villa FJ, Armas J. Community-based peer-led diabetes self-management: a randomized trial. Diabetes educ. 2009; 35(4): 641-51. http://dx.doi.org/10.1177/0145721709335006

33. Lee MC, WU SV, Hsieh NC, Tsai JM. Self-Management on eGFR, depression and Quality of life patients with Chronic Kidney Disease: A Meta-Analysis. Asian Nursing Research. 2016;10(4): 255-62. http://dx.doi.org/10.1016/j.anr.2016.04.002

34. Loring K, Ritter Pl, Pifer C, Werner P. Effectiveness of the chronic disease self- management program for persons with a serious mental illness: a translation study. Community Ment Health J. 2014; 5O(1): 96-103. http://dx.doi.org/10.1007/s10597-013-9615-5

35. Eyles C, Leydon GM, Hoffman C J, Copson ER, Prescott P, Chorozoglou M, et al. Mindfulness for the self-management of fatigue, anxiety, and depression in women with metastatic breast cancer: $\mathrm{a}$ mixed methods feasibility study. Integr Cancer Ther. 2015;14(1): 42-56.

http://dx.doi.org/10.1177/1534735414546567

36. Garnett A, Ploeg J, Markle-Reid M, Strachan PH. Self-management of multiple chronic conditions by community-dwelling older adults: A concept analysis. SAGE Open Nursing. 2017; 1: 1-16 https://doi.org/10.1177/2377960817752471 
37. Schulman-Green D, Jaser SS, Park C, Whittemore R. A Metasynthesis of factors affecting selfmanagement of chronic illness. J Adv Nurs. 2016; 72(7): 1469-89. https://doi.org/10.1111/jan.129o2

38. Sakraida TJ, Weber MT. The Relationship between depressive symptoms and self-management behaviors in patients with T2DM and Stage 3 CKD. Perspect Psychiatric Care. 2016; 52(4): 273-82. https://doi.org/10.1111/ppc.12128

39. Manaze D, Everrett B, Astorga C, Yogendra D, Salomason Y. The influence of health literacy and depression on diabetes self-management: A Cross-Sectional Study. J Diabetes Res. 2016; 2016: 3458969. http://dx.doi.org/10.1155/2016/3458969

40. Peñarrieta MI, Reyes G, Krenderdt S, Flores F, Resendiz E, Chávez-Flores E. Automanejo en enfermedades crónicas: diabetes mellitus tipo 2, hipertensión arterial y cáncer. Revista de Investigación de la Universidad Norbert Wiener. 2015; (4): 43-53. https://bit.ly/3b6xma7

41. Mar-García J, Peñarrieta-de Córdova I, León-Hernández R, Gutiérrez-Gómez T, Banda-González $\mathrm{O}$, Rangel-Torres S, et al. Relación entre automanejo y percepción de funcionalidad familiar en personas con diabetes mellitus tipo 2. Enferm Univ. 2017; 14(3): 155-61.

http://dx.doi.org/10.1016/j.reu.2017.05.005

42. León-Hernández RC, Peñarrieta-de Córdova MI, Gutiérrez-Gómez T, Banda-González O, Flores-Barrios F, Rivera MC. Predictores del comportamiento de automanejo en personas con enfermedades crónicas de Tamaulipas. Enferm. Univ. 2019; 16(2):128-37.http://dx.doi.org/10.22201/eneo.23958421e.2019.2.637

43. León HRC, Peñarrieta DCM, Gutiérrez GT. Capítulo V:Validación de instrumentos de indicadores de salud y psicosociales. Red de automanejo de enfermedades crónicas. En: Gutiérrez GT. Estrategias de aprendizaje en cronicidad. México: Colofón; 2019. p.65-84.

44. Kroenke K, Spitzer RL, Williams JB. The PHQ-9 Validity of brief depression severity measure. J. Gen Intern Med. 2001; 16(9): 606-13. https://dx.doi.org/10.1046/j.1525-1497.2001.016009606.x

45. Kroenke K, Spitzer RL. The PHQ-9: A New Depression Diagnostic and severy measure. Psychiatric Annals. 2002; 32(9): 1-7. http://dx.doi.org/10.1046/j.1525-1497.2001.016009606.x 